International Journal of Engineering \& Technology, $7(2.33)(2018) 591-596$
SPC
Website: $w$ ww.sciencepubco.com/index.php/IJET
Research paper

\title{
neck-carean early warning system to prevent neck pain
}

\author{
Rani. T. $P^{1 *}$, Shalini. $G^{2}$, Sivapriya $\mathbf{P}^{2}$, Jayashri. $\mathbf{P}^{2}$ \\ ${ }^{1}$ Associate Professor, Dept. of Information Technology, Sri Sairam Engineering College \\ ${ }^{2}$ Student, Dept. of Information Technology, Sri Sairam Engineering College \\ *Corresponding author E-mail: nishaliguna@gmail.com
}

\begin{abstract}
Technology has been playing a vital role in day-to-day life of the mankind. Eventually, it has also made a serious impact on the society by affecting the health condition of the people. A recent research proves that nearly $71 \%$ of the people community has been affected by the neck pain. The major factor for this impact is the long term usage of the mobiles or computers. As we are forced in an environment where we work for a very large time focusing upon a single position, there is risk of getting neck pain. To mitigate this problem, we proposed a sys-tem which alerts the user by tracking the neck movements. The sensor will be placed at the back of the user's head to determine the neck movements since the neck muscles are attached with the head. For detecting the neck movement, we have used MEMS SENSOR which can determine the variations in 3600degree. This sensor will be interfaced with the system via USB Cable through the arduino board. An alert message will be popped-out on the screen by the system to notify the user if the system finds slight variations in the neck movement. The data collected from sensor through data logging process will be sent to the registered mail of the user at the time of authentication. The sug-gestions will also be displayed as a pop-up message to reduce the strain. We have achieved approximately 93\% accuracy, during testing process.
\end{abstract}

Keywords: Arduino Board; MEMS SENSOR; Neck Movement; USB Cable

\section{Introduction}

Technology had seen a tremendous growth in recent. Invention of electronic gadgets has eased the work of the people. Combined with technological advancements, people are required to work for long, uncomfortable durations. This tends to increase the muscle strain which in overtime may even lead to wear and tear on spine and other parts of the body.

When we strain the neck muscles, we are making the natural cervical alignment to dismantle. This misalignment can even lead to several other problems and may even make the life span to a shorter period. Experienced surgeons suggest that if we tend to give more pressure on our neck portion, it can reduce the capacity of the lungs to $30 \%$.

The existing system uses headset to detect the neck movements and it captures the neck orientation in 3D view. Unfortunately, the systems just provided the user to view their movements in 3D view in their mobiles and it had failed to alert the users to give suggestions about their health status. Another drawback of the existing system is that the devices are very expensive.

Neck-Care helps the user in a better way as it is the tool which is compact and user friendly. The 3 -axis accelerometer sensor is used for the purpose of capturing the neck movements and issuing an alert notification to the users by determining the neck variations. The values captured are recorded in the mail for future uses.

\section{Aim of the proposed system}

The main aim of the system is to make people to get relief from the neck pain and text-neck problems and to enhance their postures to lead a comfortable life. We intend to develop a wearable device that will be available in an affordable price. With our system, the health conditions of the people can be improved as our product gives suggestions and therapies. The proposed is a sensor based system for determining the neck movements. The proposed project can support end users economically since it is developed with low cost and is affordable to any kind of users. Automatic alert notifications with suggestions and therapies are provided by system which can help the people to know about their postures to reduce the neck pain effects. Also, a graph is displayed on the screen to show the comparison of a good posture and actual posture obtained until the person wears our product.

\section{Text neck}

Text Neck is a syndrome or a repetitive stress caused due to holding our head in a forward or a backward position for long period of time [1]. Due to the excessive stress given to the neck part, tensions are created in a deep muscles of our neck and across the shoulder part. The Text Neck is shown in the Figure 1. This causes chronic neck pain. Also, chronic headaches may also occur due to this problem. Text Neck is an epidemic disease. It has been increased as the usage of mobiles, laptops or any other electronic gadgets have increased tremendously. Typically, neck pain occurs with ages. But according to the report, it has been proved that neck pain even seen with teenage. Permanent damages can also be caused due to Text Neck. The natural curvature of the neck changes when people stretch their neck either in forward direction or backward direction. 


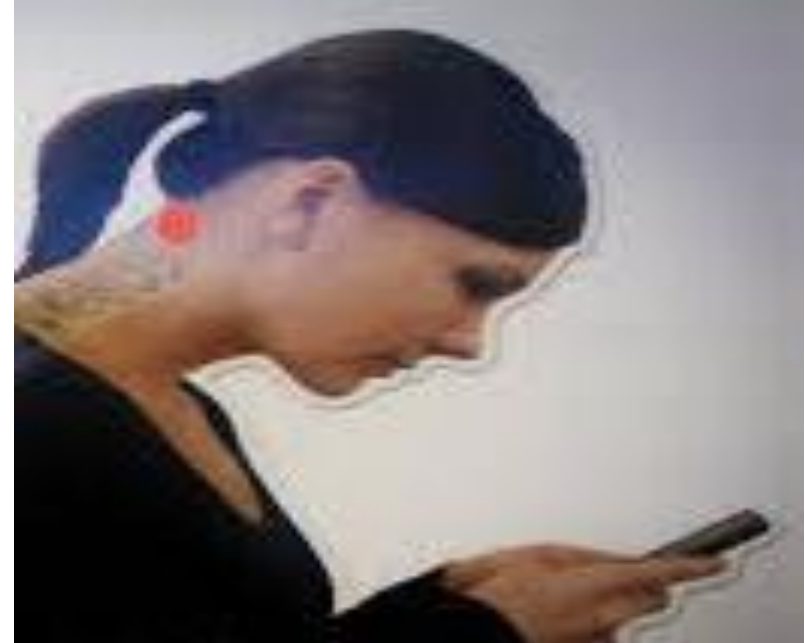

Fig. 1: Typing the Messages by Stretching the Neck to the Chest May Cause Text-Neck Which is highlighted as a Red Spot in the Image.

When people stretch their neck to their chest for a prolonged period, it truncates the breath. Eventually, leading to lung diseases. Experienced surgeons state that stressing the neck region for more hours may lead to shorten the time period of the lungs and may even shorten the life-span. Text Neck can reduce the capacity of the lungs to $31 \%$.

\subsection{Effects of bad postures}

Human head weighs approximately about 11 to 12 pounds. This weight is supported by our neck muscles. Normally, in correct posture our neck is not strained in order to support the headweight. But, when we adopt improper postures, additionally it increases the weight supported by the neck part. The increase in the neck muscles to support the head weight may vary from $22 \mathrm{lbs}$ to 40lbs. This causes huge strain in the neck part which spreads to the shoulder regions leading to several other consequences.

Also, bad posture may result in the disorder of neck angular movement[2]. Each and every degree tilt in the neck region, results in various neck angular movement. Since, the posture is displaced from the regular one, it causes changes in the curvature of the neck. The effects of change of curvature due to the tilt in the neck region is shown in the Figure 2.

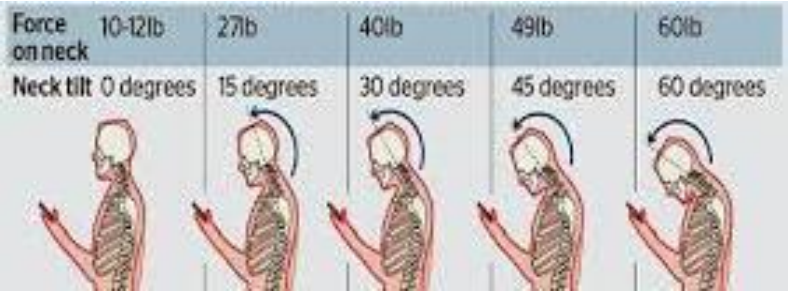

Fig. 2: Effects of Changes in the Curvature of the Neck Muscles.

Our main objective is to relegate the occurrence of the neck pain by giving an early warning to the user through the orientation of their neck postures. The sensors placed at the back of the neck senses the variations of the neck motion. The system is coded such that if the variations collected by the sensors are too slight over the given period of time, then an alert message will be popped out and will be displayed on the screen which has been interfaced with sensor via blue-tooth transmitter. These variations along with the readings will be shared to the user's mail. Suggestions and therapies will be automatically notified to the user. These suggestions and therapies will be triggered once in a week.

\section{Related works}

[3] It is a posture training app tool is composed of a wearable posture training headset. The wearable training headset is equipped with real-time sensors to monitor head and neck postures. The App displays the values collected from the sensor. In the App, Students can glance over their friends' posture performance. The application allows the student to share their report with others. This application is used only for students.

[4] In this paper, to improve the accuracy and calculation speed, the authors performed a comparison between two image processing algorithms, which involved in calculating neck angles while using the smart-phone. The two image detection algorithms were HAAR and LBP (Local Binary Patterns).Both of them had their own advantages and disadvantages. The main difference between the two algorithms was that HAAR used floating-point for the calculation, while LBP used integer numbers. The comparison showed the differences of the two algorithms in terms of accuracy and calculation speed.

[5] This work analyzed the movements and the muscular activity during motocross sessions and how a neck brace influence both. These movements are measured in terms of angles using two $\mathrm{Bi}$ ometrics ${ }^{\circledR}$ angular sensors (one for biplanar flexion and one for torsion); the muscle activity is registered using four pairs of electrodes (muscle selected: Sterno-Cleido-Mastoid left and right, Trapezium left and right). The results obtained, concluded that the pilot wants to focus the attention to the next jump or obstacle while accelerations of different nature are acting on the neck, therefore isometric contractions are predominant.

[6] This work offered a continous, non-invasive solution for assessing cranio-vertebral (CV) angle for FHP detection using inertial body sensors. The results obtained are validated against a conventional in-clinic method, the Electronics Head Posture Instrument (EHPI), demonstrating the possibility of pervasive detection of FHP. In addition, a real-time bio-feedback mechanism for postural correction and prevention is presented, and results from a pilot study show its effectiveness in reducing the amount of time subjects spend in FHP.

[7] This research monitors the posture of a person's neck and converted the user neck's posture into 3D geometrical view. The system used Least Squares Orthogonal Distance Fitting (LSODF) to measure the user neck's posture in 3D geometry. Accelerometer sensors were used to measure human neck posture. They installed this sensor into the commonly found inner Bluetooth earphone. The user wears the earphone in the ear. Then, the 3-axis signals(x, $\mathrm{y}, \mathrm{z}$ ) are generated based on the user's neck posture from various directions and angles. They are represented as points in 3D space. LSODF implements model fitting with these points in 3D space.

\section{Proposed system}

Due to the work pressure and the working environment, people are facing serious pain. To relegate from this issue the proposed system works to ensure the welfare of the society by intimating the user with the alert message. MEMS SENSOR placed at the back part of the neck portion collects the variation of the neck movements. Data collected from the sensor is sent to the user's mail. The system checks for the variations. If it finds that the variation is slight, a pop-up message will be alerting the user to take relax or have some water. To trigger the alert messages and suggestions we have kept some threshold assumptions which is discussed below. All the variations are stored in the mail server. Automatic suggestions and therapies will be triggered by the system which can be viewed in the user's screen. Also, the proposed displays a graph which compares the actual posture of the user with the good posture in order to show their health status.

\section{Technical specifications}

\subsection{MEMS sensor (3-axis accelerometer sensor)}

Micro-electro-mechanical systems is the technology of the microscopic devices, which is made up of the components which is of size 20 micrometer [8]. It is made up of several materials like 
silicon, polymers, metals and ceramics. It has been in evolution since 1960s. With the advancement in the technology it has been evolved in various fields especially it had contributed a lot in the motion detection. There are various types of MEMS SENSOR available in the market. The one which is used in our system is inertial body sensor. This inertial body sensor is classified into various types. We have used 3-Axis Accelerometer Sensor which is shown in the Figure 3. This is commonly found in almost all the smart phones today. This Accelerometer Sensor is much efficient and it is inexpensive. These sensor are used in the phones to change the orientation of the mobile screen. That is, the user can change the view to either portrait or landscape.

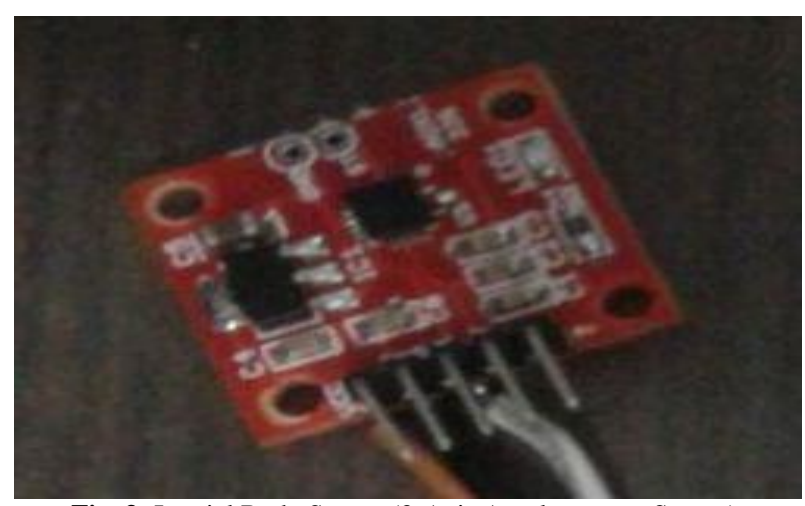

Fig. 3: Inertial Body Sensor (3-Axis Accelerometer Sensor).

\subsection{Arduino board}

Arduino is an open source hardware platform which can help in manufacturing interactive applications [9]. It senses and controls the system to provide the required output. It consists of microprocessors and microcontrollers which are programmed using either Java, $\mathrm{C}$ or $\mathrm{C}++$. here, $\mathrm{c}$ programming language is used to design the board. There are so many versions of arduino is available in the market. In our system we have used Arduino UNO R2. The UNO differs from all preceding boards in that it does not use the FTDI USB-to-Serial Driver Chip. Instead, it features the Atmega8 U2 programmed as a USB-to-Serial Converter. This arduino board is connected to the MEMS SENSOR via USB cable which is also called as connectors. It needs approximately $0.8 \mathrm{~A}$ of current which is provided when it is interfaced with the system.

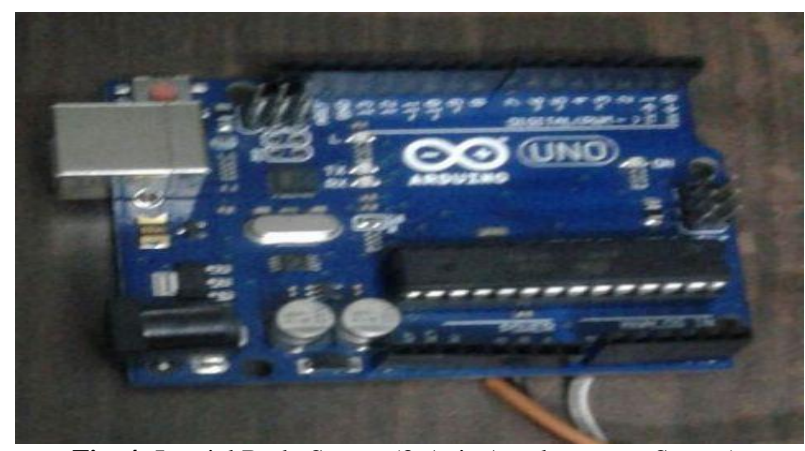

Fig. 4: Inertial Body Sensor (3-Axis Accelerometer Sensor).

\subsection{Arduino IDE}

Arduino IDE is an open source software platform which is used to build digital devices and interactive objects[10]. It makes the device to sense and control objects in real-time. It can be either coded with structured programming language (like C) or object oriented programming (like Java). It supports all the hardware and software platforms and hence it is architectural neutral.

\subsection{Net beans IDE}

Netbeans IDE is the official IDE for developing applications with java. Netbeans with its editor smoothly upgrade your applications by using code analyzers and code converters. it uses several inbuilt functions to simplify your coding. Various versions of Netbeans IDE is available. Netbeans IDE version 8 is used. Since, with its new editor, Netbeans IDE sets the standard for developing applications with cutting edge technologies [11]. An IDE is much more than a text editor. The Netbeans editor indents lines, matches words and brackets, and highlights source code syntactically and semantically. It lets you easily refactor code, with a range of handy and powerful tools, while it also provides code templates, coding tips, and code generators.

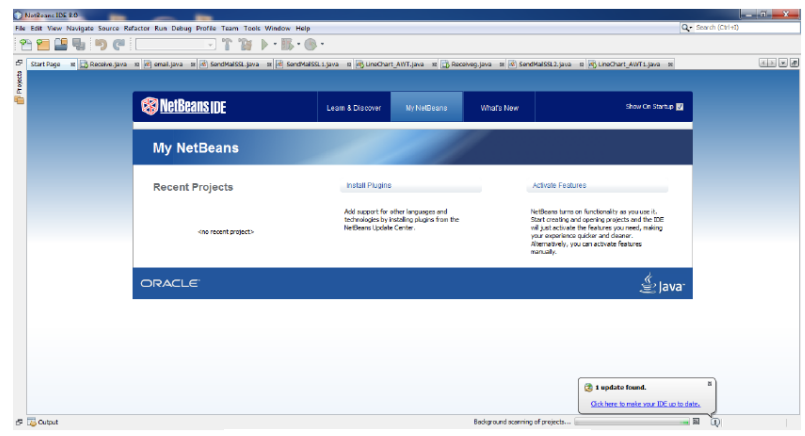

Fig. 5: NetBeans IDE Version 8.

\subsection{MySQL}

MySQL is the most popular open source relational SQL database management system. MySQL is one of the best RDBMS being used for developing various web-based software applications[12]. The MySQL Query Browser is a graphical tool designed to provide a user friendly environment in which to construct and execute SQL statements. This functionality is now provided using the MySQL workbench tool.

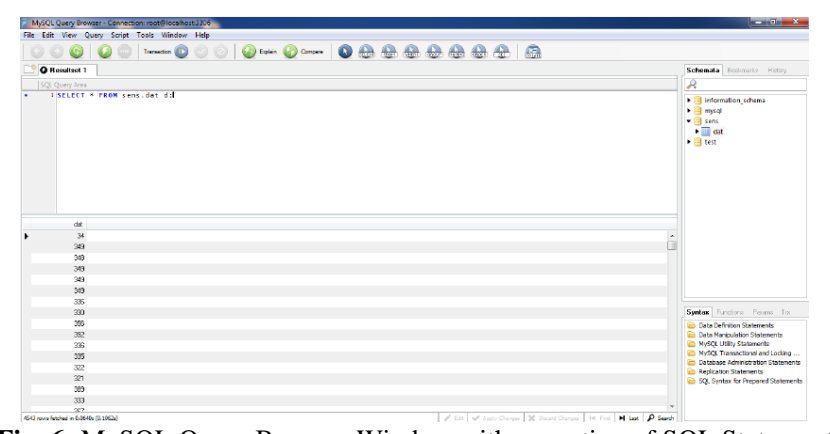

Fig. 6: MySQL Query Browser Window with execution of SQL Statement

\section{Working of the proposed system}

The MEMS SENSOR is placed at the back of the neck portion. This sensor is interfaced with the arduino board by using USB cable. This board is paired with the system using connectors. Neck movements will be captured by the sensors. Variations will be keenly noticed. If there is a slight variation, immediately an alert message will be popped-up on the screen of the device.

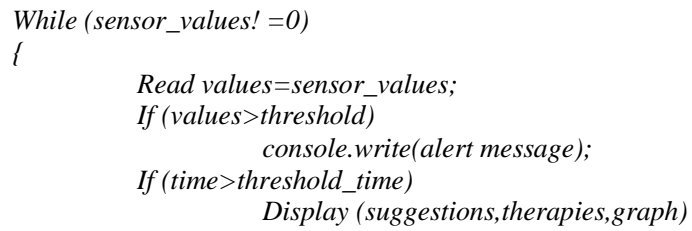

Fig. 7: Proposed System Working Logic. 


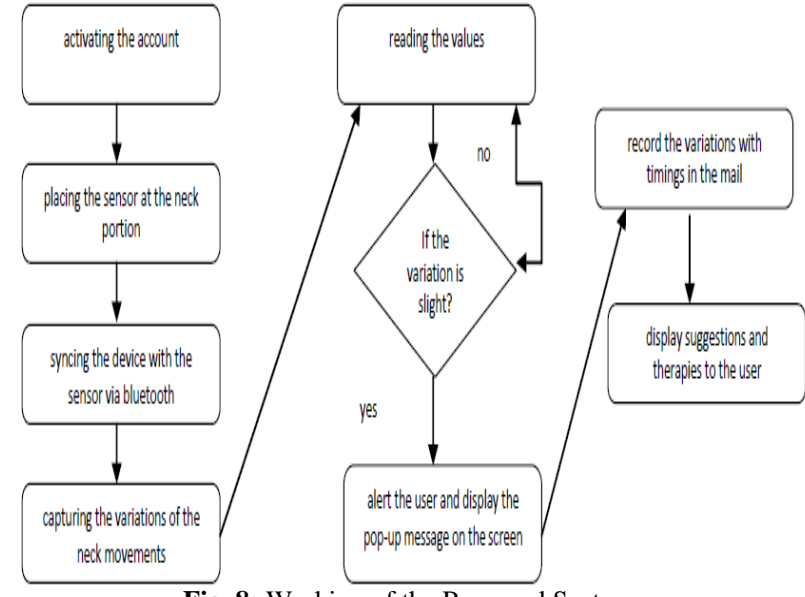

Fig. 8: Working of the Proposed System.

These variations will be recorded by the system and is shared to the user's mail which is registered during the authentication stage of the device. Suggestions are also showed as a pop-up message in the screen which helps the user to know about their health status.

\subsection{Variations and posture alerts}

In the proposed system, to alert the user we have used some threshold assumption. This alert message is given by determining the variations. The assumption of threshold is necessary because when the user works for a long time, he/she is giving more strain to the neck part which is made to support the head weight to a larger extent. The normal ranges for the neck postures is shown in the Table 1.1. So, if the normal range is not maintained by the person, an alert message has to be given.

Table 1: Normal Range Values for Various Neck

\begin{tabular}{lc}
\hline POSTURE & RANGE(in degrees) \\
\hline Flexion & $0-50$ \\
Extension & $0-60$ \\
Left Lateral Flexion & $0-45$ \\
Right Lateral Flexion & $0-45$ \\
Left Rotation & $0-80$ \\
Right Rotation & $0-80$ \\
\hline
\end{tabular}

To give this alert message, we assumed to take a time limit of ten(10)minutes. But, as per the research, if the person is working on the laptop or any other devices he/she should take a break for at least after thirty (30) minutes. Since the time constraint is little higher, we have restricted the time limit from thirty minutes to ten(10) minutes to alert the user. For displaying the suggestions, the time constraint is fifteen(15) minutes. Also the values recorded from the sensor will be sent to the user's mail at a time period of fifteen(15) minutes. Along with the suggestion message, a graph will also be triggered which compares the normal neck posture with the neck posture obtained from the user's motion.

\subsection{Suggestions and therapies}

In the proposed system, to make the user to get relief from the neck pain some suggestions and therapies are given. Those suggestions are:

1) Take a break

2) Have some water

3) Make some movements

4) Do some exercise

These suggestions make the user to take some relaxation in a hectic work pressure. The suggestion will be displayed on the screen which is paired with the sensors connected at the back portion of the neck through blue-tooth module. Also, these suggestions are stored in the user's mail and can be viewed again for later use.

Therapies is the method or a process which intends to relieve or heal a disorder[13]. Here, therapies means the process of getting reliefs from the neck pain. Our system consists of ten plus exercise moves which ease the pain. Some of the exercise suggested by our system is as shown in the Figure 9.
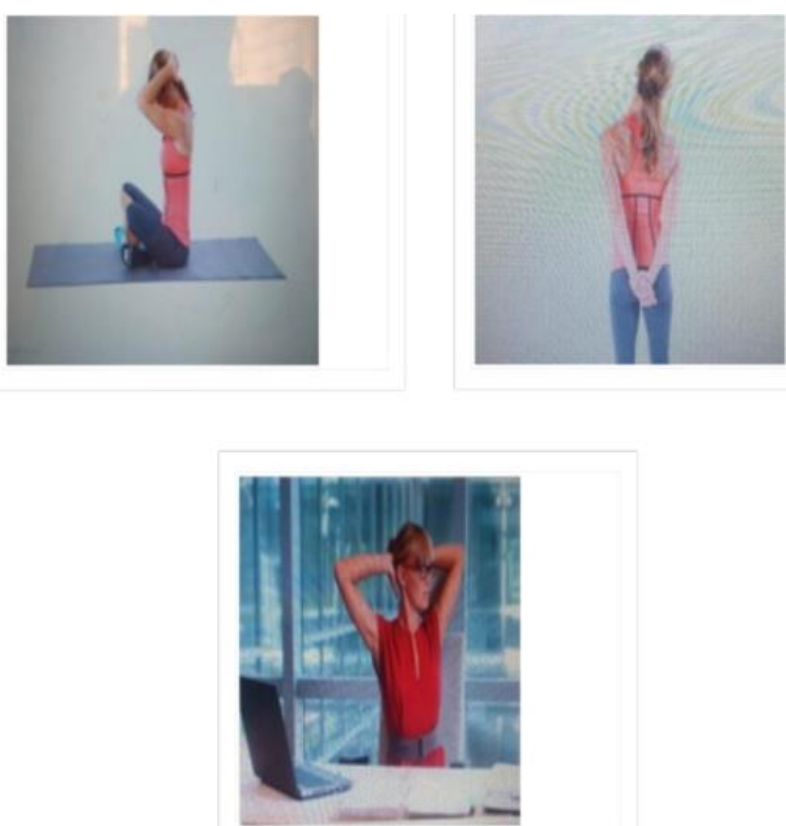

Fig. 9: Therapies Suggested by the Proposed System.

\section{Results and discussions}

The results of the proposed system which is carried with a group of three teenagers who are named as I, II, III respectively. Our objectives in the design of experiments have four folds. First is to validate the effectiveness of the developed posture detection system; secondly, to compare the good posture versus actual posture of the user. At the third stage the effect on displaying suggestions and therapies are analyzed and final step is to check that the values are send to the mail in a secure manner.

The proposed system experiment setup is done through the following steps:

Step 1: Setting up the device

This is the initial step where the sensor is interconnected with the system via USB cables. The sensor is placed at the back portion of the user as shown in the Figure 10.

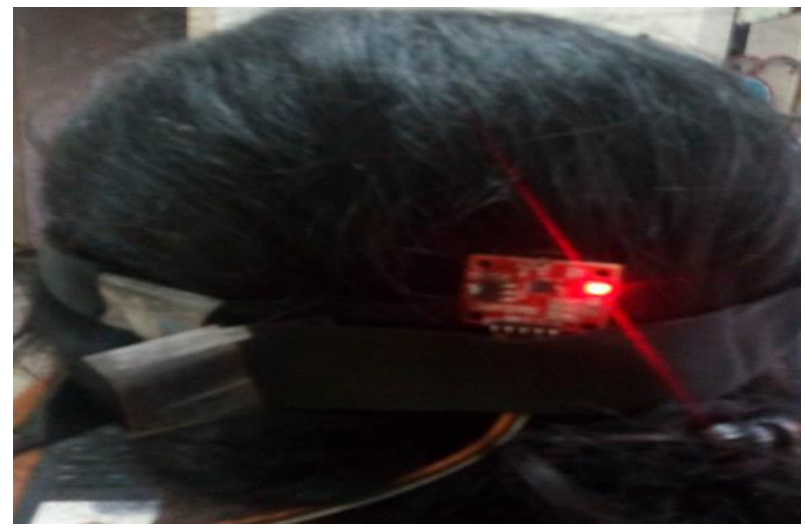

Fig. 10: Placing the Sensor at the Back Part of the Head

Step 2: Capturing the values

As the user starts to rotate the head, the orientation of the neck changes. The changes are captured by the sensor. The variations are displayed as values on the user screen as shown in the Figure 11 . 

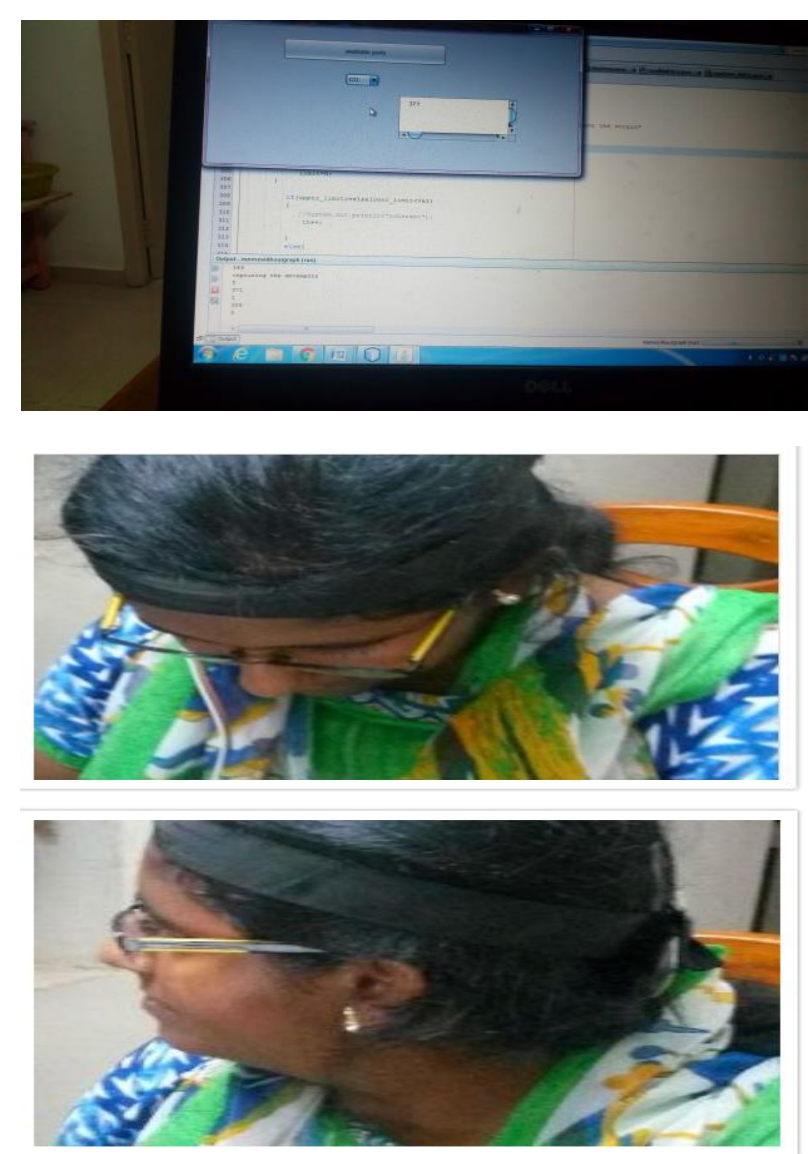

Fig. 11: Head Rotation Along with the Values.

Step 3: Issuing An Alert Notification

As the user starts to rotate the head, the orientation of the neck changes. The changes are captured by the sensor. The variations are displayed as values on the user screen as shown in the Figure 12 .

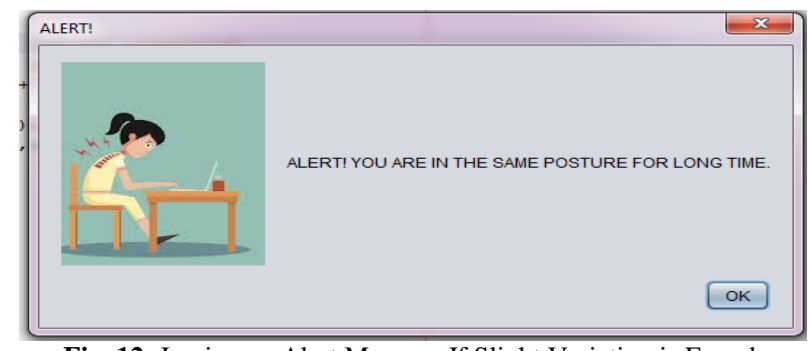

Fig. 12: Issuing an Alert Message If Slight Variation is Found.

Step 4: Displaying Suggestions and Therapies

For a particular period of time, the system tends to capture the readings. When the threshold period gets exceeded, then automatically a suggestion message along with the exercises will be displayed on the screen. Figure 13 shows the suggestion message with exercise.

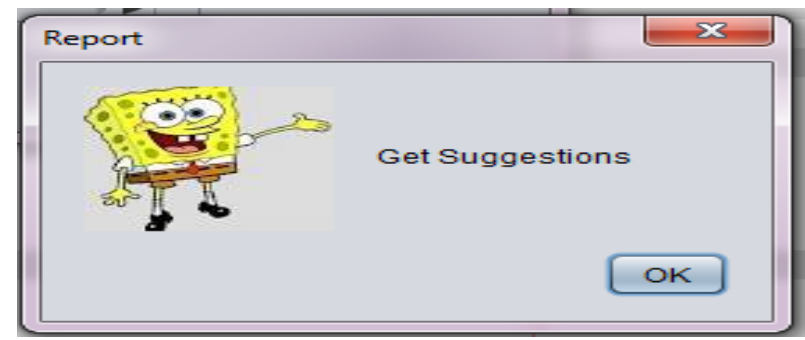

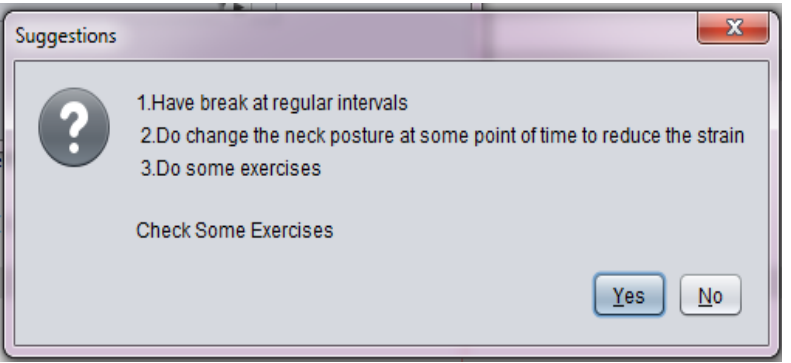

Fig. 13: Displaying Suggestions for Reducing the Impact of Neck Related Problems.

Step 5: Plotting the graph

After the values are recorded until a threshold period, it gets stored in the database through which the graph is plotted. Plotting the graph gives a comparison to the user about their postures. From that comparison, the user can get to know their health status. Comparison of the graph is shown in the Figure 14.
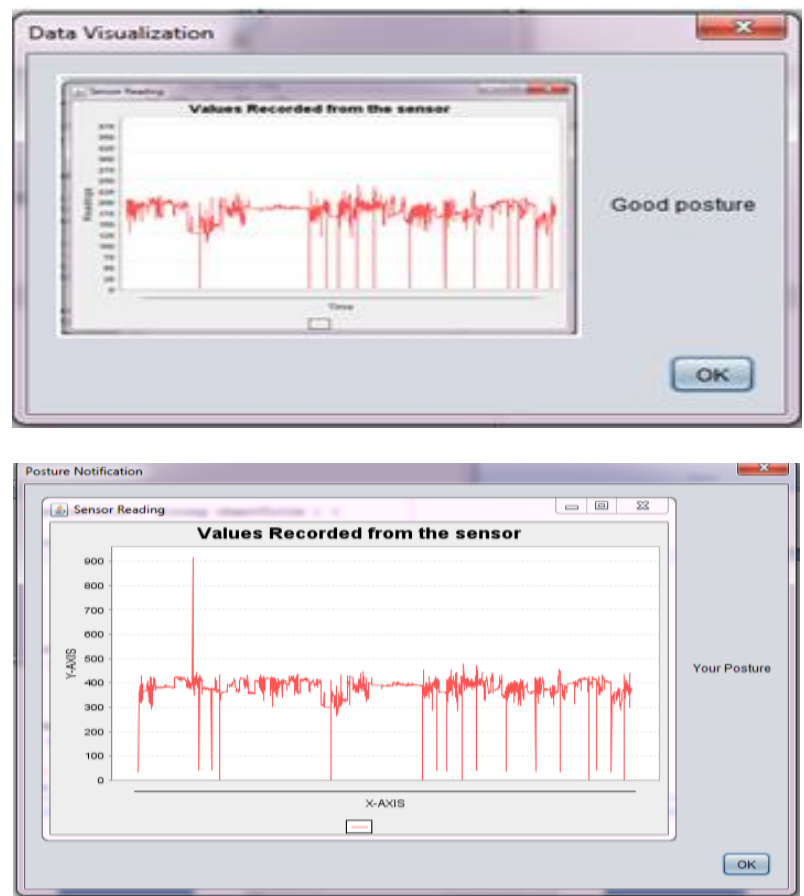

Fig. 14: Comparison between the Good Postures Versus Actual Posture

The effectiveness of the posture detection system and the comparison of postures are deliberately reviewed and validated throughout the experiments. Table 2 shows the experiment results of the values obtained from the proposed system.

Table 2: Experiment Results of the Proposed System

\begin{tabular}{lll}
\hline I & II & III \\
\hline 354 & 401 & 389 \\
374 & 324 & 397 \\
358 & 347 & 312 \\
366 & 312 & 241 \\
386 & 379 & 124 \\
\hline
\end{tabular}

From the results obtained, it is clear that the proposed system achieved better efficiency and have detected the posture accurately. Also, from the suggestions and therapies the user can improve their neck postures which thereby helps them to reduce the effect of neck pain to a considerable amount.

\section{Conclusion}

Technology has always assisted the mankind to improve the living conditions of the people. It had simplified our work to a great extent. Usage of cell phones and PDAs has being tremendously rising due to the great response from the people. But, according to 
the research it has been proved that nearly $79 \%$ of the population between the ages 18 and 44 have been affected by the text neck due to the prolonged usage of the mobiles and other electronic gadgets. This is expected to increase in coming years. To mitigate this problem, the proposed system "NECK-CARE" can serve as a best remedy to the society. Suggestions and the therapies provided by the system have been an efficient initiative to minimize this effect among the working society. In near future, we hope to develop the features like gaming which utilizes our neck postures to move the objects on the screen. The gaming feature can be made possible by utilizing the neck gestures to move the objects on the screen.

\section{References}

[1] Jason M. Cuellar, Todd H. Lanman, "Text Neck: an epidemic of the modern era of cell phones", The Spine Journal, vol. 17, no. 6, pp. 901-902, June 2017.

[2] L. Staker, J. Coleman, R. Stross, "A comparison on posture and muscle activity during tablet computer, desktop computer and paper use", Journal of the Chartered Ergonomics, vol. 10, no.3, pp 540555, June 2008.

[3] Da-Yin Liao," Collaborative, social-networked posture training (cspt) through head-and-neck posture monitoring", International Conference on Enterprise Information Systems (ICEIS 2017), Scitepress, vol. 3, pages 158-165, August 2017.

[4] Worawat Lawanont, Pornchai Mongkolnam, Chakarida Nukoolkit, Masahiro Inoue,"A comparison between two image detection algorithms on neck angle detection and a prolonged usage classification concept", South East Asian Technical University Consortium Symposium, Journal of the Chartered Ergonomics, vol. 10, pages 142150,2016

[5] Luca Gorasso, Nicola Petrone,"On-track measurements of neck movements and muscle activity during motocross sessions with or without neck brace", Asia-Pacific Congress on Sports Technology (APCST), Procedia Engineering , vol. 6, pages 337 - 342, July 2013.

[6] Taeyoung Kim, "Detection and prevention of forward head posture", International Journal of Autonomous and Adaptive Communications Systems, Body Sensor Network(BSN) IEEE Explore, vol. 15, pages $125-126$, July 2011 .

[7] Soonmook Jeong, Taehoun Song, Hyungmin Kim, Miyoung Kang ,"Human neck's posture measurement using a 3-axis accelerometer sensor", International Conference on Computational Science and Its Applications, ScienceDirect, vol. 29, pages 553-560, 2011.

[8] Kazusuke Maenaka, "Current MEMS technology and MEMS sensors -focusing on inertial sensors", International Conference on Solid State and Integrated-Circuit Technology, vol.10, no. 25, pp 448451, December 2008.

[9] K.V.S.S. Sairam, N. Gunasekaran, " Arduino UNOR2: It's function ", International Conference on Wireless Communication, vol.40, issue 6, June 2002, Available online: https://arduinounor2.zendesk.com/entries/59399217 - function.

[10] Venkatesh Neelapala, Dr. S. Malarvizhi, "Environment Monitoring System Based on Wireless Sensor Networks Using Open Source Hardware", International Journal Of Engineering Research and Sports Science, vol.2, issue 4, April 2015, available online: http://www.ijerss.com/preview.php?id=U1VwRIVsTIRMVEUwT WpneU5UZ3pPRFU9.

[11] Rakesh Sharma, Sonymol Koshy, "Promoting Open Source Technology in Education: Netbeans : The Perfect Open Source IDE", International Journal of Computer Science and Technology, vol. 2, issue 3, pp 345-368, September 2011.

[12] Dipina Damodraran, Shirin Salim, Surekha Marium Vargese, "Performing Evaluation of MySQL and MongoDB", International Journal on Cybernetics \& Informatics(IJCI), vol. 5, issue 2, pp 123-134, April 2016.

[13] Ling Jun Kong, Hong Sheng Zhan, "Therapy for Neck and Shoulder pain: A systematic review and meta-analysis", Evid Based Complement Alternat Med, vol. 30, issue 7 pp 432-440, Feb 2013 